\title{
Verifying stabilizing controllers for performance improvement using closed-loop data
}

\author{
Sung H. Cha ${ }^{1, *, \dagger, \ddagger}$, Arvin Dehghani ${ }^{1, \ddagger}$, Weitian Chen ${ }^{1, \ddagger}$ and Brian D. O. Anderson ${ }^{1,2}$ \\ ${ }^{1}$ Research School of Engineering, The Australian National University, Canberra ACT 0200, Australia \\ ${ }^{2}$ National ICT Australia Ltd., Locked Bag 8001, Canberra ACT 2601, Australia
}

\begin{abstract}
SUMMARY
In iterative identification and control, one needs to determine the closed-loop performance of a new controller designed with the current model so that a decision can be made on whether a new iteration of plant identification and controller design is necessary. In multiple model adaptive control, at times, a decision to connect a different controller is made. It is desirable to check the closed-loop performance with a new controller before it is switched in. Consider a feedback interconnection of an unknown linear plant and a known linear stabilizing controller, and assume that some knowledge of the closed-loop system is available. We aim to develop theory and tools for experimentally verifying the closed-loop performance with a prospective stabilizing controller in advance. The simulation results demonstrate efficacy and versatility of our results. Copyright (C) 2013 John Wiley \& Sons, Ltd.
\end{abstract}

Received 24 July 2010; Revised 31 August 2012; Accepted 28 January 2013

KEY WORDS: closed-loop performance; controller performance verification; iterative identification and control

\section{INTRODUCTION}

In iterative identification and control (see, e.g., [1-5]), one often starts with a rough model of the plant and then designs a controller on the basis of this model hoping to achieve certain closed-loop performance. If the designed controller cannot achieve the desired closed-loop performance, then one will re-identify the plant to obtain a better model and design a new controller on the basis of this new model. If the new controller is capable of achieving the desired goals, then the iteration will stop. Otherwise, a new iteration of plant identification and controller design is needed until a satisfactory controller (if there exists one) is found. In multiple model adaptive control (MMAC) (see, e.g., [6-12]), based on measurements with the existing controller, one may decide to replace the controller by a different one drawn from a prescribed set.

An important task in the iterative identification and control or MMAC procedures is to check if the controller designed on the basis of the current model can achieve the desired closed-loop performance with the given plant. When an indication of the performance degradation occurs, the supervisory mechanism suggests the need to collect more data to obtain an improved model via identification or to start a test procedure leading to controller replacement. This is a nontrivial task. In fact, many multicontroller adaptive switching algorithms do not explicitly rule out the possibility of placing a destabilizing controller in the closed-loop [11,13]. Even if the new controller is ensured to be stabilizing, it is not straightforward to verify whether the new controller will satisfy all the performance requirements and guarantee a better performing closed-loop.

\footnotetext{
*Correspondence to: Sung H. Cha, AgJunction, PO Box 3865, South Brisbane QLD 4101 Australia.

†E-mail: u3951656@anu.edu.au

$¥$ The work of this paper was done when these authors were with the Research School of Engineering, The Australian National University, Canberra ACT 0200, Australia.
} 
Verifying analytically the closed-loop performance of a controller in advance without inserting it into the control loop is a nontrivial task if not an impossible one in practice [13, 14]. The fundamental difficulty is that the plant involved may be unknown or most commonly only partially known [15-17]; such knowledge as is available is shaped by the existing controller. One should note that as no accurate plant model(s) may be available for the offline (or analytical) tests, some form of online (or experimental) tests can be considered for the closed-loop performance verification purposes. Although many adaptive control schemes indeed utilize the real-time measurement data to evaluate performance of the current and candidate controllers, the available closed-loop signals are most likely subject to noise disturbances, and even with a low noise, they may become inadequate to predict performance in advance [18]. Indeed, there is no indication-at least to the point of verifying user-specific performance requirements - of research attempts to evaluate the closedloop performance (as opposed to just stability, see [19]) before a new controller is inserted. In this paper, which builds on our earlier conference paper [20] that was largely confined to scalar plants and incomplete in a number of aspects, we shall propose novel analysis tools for experimentally verifying the closed-loop performance of a proposed stabilizing controller. In particular, we aim to utilize a limited amount of noisy input-output experimental data obtained from a stable closed-loop system to determine aspects of the closed-loop performance of the new controller.

The structure of the paper is as follows. Section 2 states the problem of interest and reviews definitions and notations from the relevant literature that will be used. Section 3 presents our main results for verifying closed-loop performance on the basis of the proposed experimental setting from [19]. Two examples are provided in Section 4. The first example is to show how to use a particular transfer function to verify closed-loop performance using a simple plant and controllers. The second example is a flexible-link robot arm plant, which is used to illustrate how the proposed closed-loop performance results can be applied as effective tools in an iterative identification and control strategy called the windsurfer approach to detect if a new iteration of model identification and controller design is needed through checking the closed-loop performance of a newly introduced controller before its insertion into the loop. Section 5 contains concluding remarks and future research directions.

\section{PROBLEM FORMULATION AND PRELIMINARIES}

In this section, we shall formulate the problem of interest and provide definitions and notations that will be used.

\subsection{Problem of interest}

We consider a common scenario of iterative identification and controller redesign, or MMAC algorithms, where an unknown —or only partially known-linear plant $P$ is initially operating with a known controller $C_{0}$ as a stable closed-loop, and the introduction of a new controller $C_{1}$ might be attractive. However, before we actually insert $C_{1}$ into the control loop, we would like to have an assurance that it will not cause any closed-loop performance problems. Our major interest is to develop analysis tools so that practical experimental tests can be carried out to offer such an assurance.

More formally, our problem of interest is formulated as follows: Let $\left[P, C_{0}\right]$ denote a feedback control interconnection consisting of a plant $P$ and a stabilizing controller $C_{0}$. Further, assume that the transfer function $P(s)$ is unknown, whereas $C_{0}$ is known. Let $C_{1}$ be a new controller designed to replace $C_{0}$. Now, the question is: can one develop tests to verify if $C_{1}$ in place of $C_{0}$ will result in better closed-loop performance on the basis of the knowledge of $C_{0}(s)$ and $C_{1}(s)$ and on data obtained from experiments on the closed-loop $\left[P, C_{0}\right]$, but not directly on $P(s)$ ?

Two key remarks follow:

\section{Remark 1}

It is required that a stabilizing controller $C_{0}$ is known a priori for the given unknown plant. This might appear to be quite demanding. However, it is very often true that a known stabilizing controller 


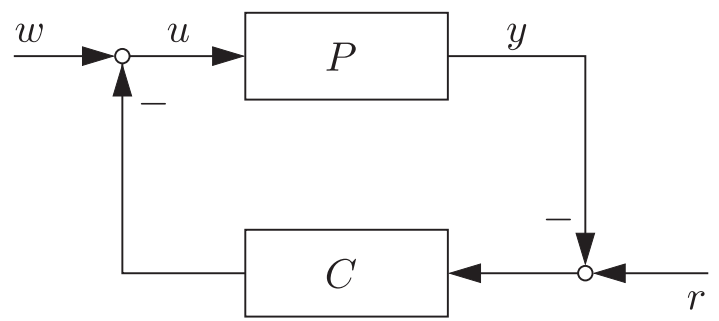

Figure 1. Standard feedback configuration.

is actually available for many practical control systems; for example, many industrial processes are often stably controlled by known PID controllers. Moreover, in iterative identification and control, it is also normal that one will commence with a known stabilizing controller.

\section{Remark 2}

If one already has a known stabilizing controller, then why does one need a new controller? There are several reasons. First, the known stabilizing controller may not offer satisfactory closed-loop performance. Second, the controller may not be able to meet new performance requirements that are more aggressive or demanding, although it meets initially proposed requirements. Finally, the plant under control might be slow-changing because of aging and/or wearing so that the known controller might no longer be able to offer satisfactory closed-loop performance as time goes on.

\subsection{Preliminaries and notations}

In this subsection, we review some notations and relevant definitions that will be frequently used in this paper, which are mostly borrowed from [21,22] and [23].

We denote by $\mathscr{H}_{2}$ the vector space of matrix-valued functions $F(s)$ analytic in the open right-half plane (i.e. $\operatorname{Re}(s)>0$ ) satisfying

$$
\|F\|_{2}:=\sup _{\sigma>0}\left(\frac{1}{2 \pi} \int_{-\infty}^{\infty} \operatorname{Trace}\left[F^{*}(\sigma+j \omega) F(\sigma+j \omega)\right] \mathrm{d} \omega\right)^{1 / 2}<\infty .
$$

Also, $\mathscr{H}_{\infty}$ denotes the space of matrix-valued functions $F(s)$ bounded in the open right-half plane such that $\|F\|_{\infty}:=\sup _{\omega \in \mathbb{R}} \bar{\sigma}[F(j \omega)]<\infty$, where $\bar{\sigma}(F)$ denotes the largest singular value of $F(s)$. We denote by $\mathscr{R}$ the set of real rational transfer functions and hence the real rational subspace of $\mathscr{H}_{2}$ (respectively, $\mathscr{H}_{\infty}$ ) by $\mathscr{R}_{\mathscr{H}_{2}}$ (respectively, $\mathscr{R} \mathscr{H}_{\infty}$ ).

Let $P \in \mathscr{R}$ be the given plant and $C \in \mathscr{R}$ be the controller, and throughout this paper, $P$ and $C$ are assumed to be proper and rational and of compatible dimension. Then, we denote by $[P, C]$ a common configuration of feedback interconnection ${ }^{\S}$ as shown in Figure 1.

Definition 3 ([21])

The interconnection $[P, C]$ of Figure 1 is said to be well-posed if the mapping $H_{[P, C]}:\left(\begin{array}{l}r \\ w\end{array}\right) \mapsto\left(\begin{array}{l}y \\ u\end{array}\right)$ exists. Furthermore, this interconnection is said to be internally stable if it is well-posed and $H_{[P, C]} \in \mathscr{R} \mathscr{H}_{\infty}$.

If $[P, C]$ in Figure 1 is well-posed, one can express $H_{[P, C]}:\left(\begin{array}{l}r \\ w\end{array}\right) \mapsto\left(\begin{array}{l}y \\ u\end{array}\right)$ as

$$
H_{[P, C]}:=\left[\begin{array}{c}
P \\
I
\end{array}\right](I-C P)^{-1}\left[\begin{array}{ll}
-C & I
\end{array}\right]=\left[\begin{array}{cc}
-P(I-C P)^{-1} C & P(I-C P)^{-1} \\
-(I-C P)^{-1} C & (I-C P)^{-1}
\end{array}\right] .
$$

Throughout this paper, we assume the physical control system is implemented as in Figure 1. Our major interest is to check the performance of a controller $C$ without inserting it into the actual control loop through carrying out experiments on a related experimental setting that is shown later in Figure 2.

\footnotetext{
${ }^{\S}$ The use of two negative-signed interconnections is adopted from [23] for ease of utilizing their results.
} 


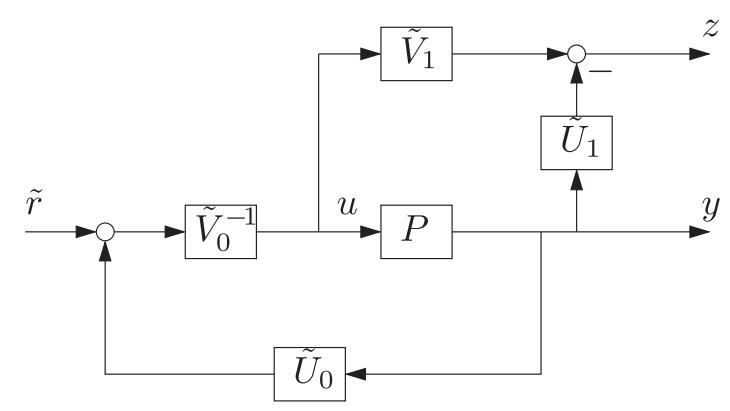

Figure 2. Experiment setting: $C_{0}=\tilde{V}_{0}^{-1} \tilde{U}_{0}, C_{1}=\tilde{V}_{1}^{-1} \tilde{U}_{1}$.

The closed-loop performance with the controller $C$ is determined by the four transfer functions defined by the four entries of $H_{[P, C]}$. For example, because $y=-P(I-C P)^{-1} C r+P(I-$ $C P)^{-1} w$ and $u=-(I-C P)^{-1} C r+(I-C P)^{-1} w$, for a good disturbance rejection for the disturbance $w$, it is desired to have $\bar{\sigma}\left(P(I-C P)^{-1}\right)$ and $\bar{\sigma}\left((I-C P)^{-1}\right)$ to be sufficiently small over the frequency range of interest. If $r$ is the reference signal, then it is desirable to have $\bar{\sigma}\left(-P(I-C P)^{-1} C\right) \approx 1$ at the frequencies of $r$. In this paper, by closed-loop performance, we always mean those performance measures related to some or all of the four transfer functions in $H_{[P, C]}$. If any of the four transfer functions does not behave well, then it will certainly indicate a performance problem.

Definition $4([19,23])$

The ordered pair $(N, M)$, with $M, N \in \mathscr{R} \mathscr{H}_{\infty}$, is a right-coprime factorization (rcf) of $P \in \mathscr{R}$ if $M$ is invertible in $\mathscr{R}, P=N M^{-1}$, and $N$ and $M$ are the right-coprime over $\mathscr{R} \mathscr{H} \infty$. Furthermore, the ordered pair $(N, M)$ is a normalized $r c f$ of $P$ if $(N, M)$ is an $\operatorname{rcf}$ of $P$ and $M^{*} M+N^{*} N=I$.

Definition $5([19,23])$

The ordered pair $(\tilde{V}, \tilde{U})$, with $\tilde{V}, \tilde{U} \in \mathscr{R} \mathscr{H}_{\infty}$, is a left-coprime factorization (lcf) of $C \in \mathscr{R}$ if $\tilde{V}$ is invertible in $\mathscr{R}, C=\tilde{V}^{-1} \tilde{U}$, and $\tilde{V}$ and $\tilde{U}$ are the left-coprime over $\mathscr{R} \mathscr{H}_{\infty}$. Furthermore, the ordered pair $(\tilde{V}, \tilde{U})$ is a normalized lcf of $C$ if $(\tilde{V}, \tilde{U})$ is an lcf of $C$ and $\tilde{V} \tilde{V}^{*}+\tilde{U} \tilde{U}^{*}=I$.

Let $(N, M)$ be an $\operatorname{rcf}$ of $P$ and $(\tilde{V}, \tilde{U})$ an lcf of $C$, and define

$$
G:=\left[\begin{array}{c}
N \\
M
\end{array}\right], \quad \tilde{K}:=\left[\begin{array}{cc}
-\tilde{U} & \tilde{V}
\end{array}\right]
$$

to be the graph symbol of $P$ and the inverse graph symbol of $C$, respectively.

The following definitions of two closed-loop performance measures are taken from [23].

Definition 6

For $P \in \mathbb{C}^{p \times q}$ and $C \in \mathbb{C}^{q \times p}$, define

$$
\rho(P, C, \omega)=1 / \bar{\sigma}\left(H_{[P, C]}\right)(j \omega),
$$

and

$$
b_{[P, C]}:= \begin{cases}\left\|H_{[P, C]}\right\|_{\infty}^{-1}, & \text { if }[P, C] \text { is stable } \\ 0, & \text { otherwise. }\end{cases}
$$

If $[P, C]$ is stable, it follows from the aforementioned definition that

$$
b_{[P, C]}=\inf _{\omega} \rho(P, C, \omega) .
$$


A $b_{[P, C]}$ of a value very close to zero (i.e. very large $\left\|H_{[P, C]}\right\|_{\infty}$ ) means that there exists very high gain in at least one of the four transfer functions in $H_{[P, C]}$ in some certain frequency range. Such large gain usually indicates a closed-loop performance problem; hence, the value of $b_{[P, C]}$ can serve as a good indication of the closed-loop performance.

\section{ANALYSIS AND EXPERIMENTAL SETTING}

In this section, we shall provide our main results on closed-loop performance verification through experiments on an experimental setting shown in Figure 2, which was initially proposed in $[12,19]$ to test the stability of a new controller. This will be achieved by revealing the fact that all transfer functions in $H_{\left[P, C_{1}\right]}$ corresponding to Figure 1 (with $C$ being replaced by $C_{1}$ ) can be determined by three transfer functions (which will be defined later) in Figure 2. This justifies why Figure 2 can be used to test the performance of a controller implemented as in Figure 1.

One should note that coprime factors of $C_{0}$ in Figure 2 are implemented in a special formreferred to as the 'Observer-form implementation'-where two left coprime factors of $C_{0}, \tilde{V}_{0}^{-1}$ and $\tilde{U}_{0}$, are separately placed in the feedforward path and feedback path, respectively. With such implementations, one can avoid the restrictions imposed by the poles and zeros of the controller on the response from $\tilde{r}$ to $y$ (see Chapter 5 in [23] and [24] for more details). It should be pointed out that there is no need to split the physical controller $C_{0}$ in real experiments because Figure 2 can be implemented with an equivalent setting, see [19] for details. Also, in case $C_{0}$ is open-loop stable, which is often the case, one can take $\tilde{V}_{0}=I, \tilde{U}_{0}=C_{0}$ and Figure 2 becomes identical with Figure 1; see [24] for details.

To predict the internal stability of $\left[P, C_{1}\right]$ before the insertion of $C_{1}$ into the loop, we introduced validation tests in $[12,19]$ utilizing a limited amount of experimental data obtained from the stable closed-loop interconnection $\left[P, C_{0}\right]$. Our earlier results propose a type of phase test analogous to the Nyquist criterion and utilize the noisy frequency response information of the closed-loop mapping $T: r \rightarrow z$ in Figure 2 to check if $C_{1}$ will stabilize the unknown plant $\mathrm{P}$. This was all achieved despite the restrictive assumption that no a priori information about the plant was available. This aimed to address the so-called transient instability problem in the context of MMAC and iterative identification and control ideas discussed in Section 1. We shall outline the results in $[12,19]$ (applicable to the MIMO case) for ease of reference.

\section{Theorem 7}

Let $\left[P, C_{0}\right]$ be internally stable. Let $C_{0}=\tilde{V}_{0}^{-1} \tilde{U}_{0}$ and $C_{1}=\tilde{V}_{1}^{-1} \tilde{U}_{1}$ be left coprime factorizations over $\mathscr{R} \mathscr{H}_{\infty}$. Consider the configuration in Figure 2 and define the mapping $T: \tilde{r} \mapsto z$ to be

$$
T=\left[\begin{array}{ll}
-\tilde{U}_{1} & \tilde{V}_{1}
\end{array}\right]\left[\begin{array}{r}
P\left(I-C_{0} P\right)^{-1} \\
\left(I-C_{0} P\right)^{-1}
\end{array}\right] \tilde{V}_{0}^{-1} .
$$

Then, the following are equivalent:

(i) $\left[P, C_{1}\right]$ is internally stable;

(ii) $T^{-1} \in \mathscr{R} \mathscr{H}_{\infty}$;

(iii) $\operatorname{det} T(j \omega) \neq 0 \forall \omega$ and $\mathrm{wno}^{\mathbb{I l}} \operatorname{det} T=0$;

(iv) $\operatorname{det} T(j \omega) \neq 0 \forall \omega$ and unwarg" $\operatorname{det} T(j \infty)=\operatorname{unwarg} \operatorname{det} T(j 0)$.

\footnotetext{
${ }^{\mathbb{I}}$ The winding number wno $X$ is the number of integer multiples of $2 \pi$ by which the argument of $X(j \omega)$ changes as $\omega$ moves from $-\infty$ to $\infty$, under the assumption that $X$ is nonzero for all $\omega$.

"The unwrapped phase is a continuous function of $\omega$ derived from the original phase function by removing the discontinuities of $2 \pi$. The process of removing the discontinuities is called unwrapping the phase [25].
} 
Recall that the closed-loop performance with the controller $C$ is determined by the four transfer functions in $H_{[P, C]}$. Hence, to make use of an experimental setting shown in Figure 2 to infer conclusions on the closed-loop performance of the controller $C_{1}$, we shall first provide connections between the four transfer functions in $H_{\left[P, C_{1}\right]}$ and the transfer functions in the experimental setting in Figure 2.

\subsection{Connection between transfer functions in $H_{\left[P, C_{1}\right]}$ and Figure 2}

Note that (6) can be expressed in terms of $G$ and $\tilde{K}_{i}$ of (2):

$$
T=\left[\begin{array}{ll}
-\tilde{U}_{1} & \tilde{V}_{1}
\end{array}\right]\left[\begin{array}{r}
P\left(I-C_{0} P\right)^{-1} \\
\left(I-C_{0} P\right)^{-1}
\end{array}\right] \tilde{V}_{0}^{-1}=\tilde{K}_{1} G\left(\tilde{K}_{0} G\right)^{-1} .
$$

The mapping $T$ can be also expressed as

$$
T=\left(\tilde{K}_{1} G\right)\left(\tilde{K}_{0} G\right)^{-1}=\left[\tilde{V}_{1}\left(I-C_{1} P\right) M\right]\left[M^{-1}\left(I-C_{0} P\right)^{-1} \tilde{V}_{0}^{-1}\right]=W_{1}^{-1} W_{0},
$$

where $W_{i}: \tilde{r}(t) \mapsto u(t)$ denotes the reference-to-input transfer function, defined as $W_{i}:=$ $M\left(\tilde{K}_{i} G\right)^{-1}=\left(I-C_{i} P\right)^{-1} \tilde{V}_{i}^{-1}$.

On the basis of Theorem 7 , one has the following result.

\section{Lemma 8}

Suppose the hypotheses of Theorem 7 hold, and consider the setting in Figure 2. Let $W_{i}=$ $M\left(\tilde{K}_{i} G\right)^{-1}=\left(I-C_{i} P\right)^{-1} \tilde{V}_{i}^{-1}$. Then,

1. $W_{1}=W_{0} T^{-1} \in \mathscr{R} \mathscr{H}_{\infty}$ if $T^{-1} \in \mathscr{R} \mathscr{H}_{\infty}$.

2. $W_{1}=W_{0} T^{-1} \in \mathscr{R} \mathscr{H}_{\infty}$ implies $T^{-1} \in \mathscr{R} \mathscr{H}_{\infty}$ if the plant is open-loop stable.

Proof

Given that $\left[P, C_{0}\right]$ is internally stable, that is, $\left(\tilde{K}_{0} G\right)^{-1} \in \mathscr{R} \mathscr{H}_{\infty}$, and $M \in \mathscr{R} \mathscr{H}_{\infty}$ (by definition), $W_{0}=M\left(\tilde{K}_{0} G\right)^{-1} \in \mathscr{R} \mathscr{H}_{\infty}$. Then, if $T^{-1} \in \mathscr{R} \mathscr{H}_{\infty}$, then we have $W_{1}=W_{0} T^{-1} \in \mathscr{R} \mathscr{H}_{\infty}$.

Now, suppose the plant is open-loop stable, that is, $M^{-1} \in \mathscr{R} \mathscr{H}_{\infty}$. Then, $W_{0}=M\left(\tilde{K}_{0} G\right)^{-1} \in$ $\mathscr{R} \mathscr{H}_{\infty}$ implies $W_{0}^{-1}=\left(\tilde{K}_{0} G\right) M^{-1} \in \mathscr{R} \mathscr{H}_{\infty}$. Because $T^{-1}=W_{0}^{-1} W_{1}$, then $W_{1} \in \mathscr{R} \mathscr{H}_{\infty}$ implies $T^{-1} \in \mathscr{R} \mathscr{H}_{\infty}$.

When $C$ in Figure 1 is replaced by $C_{1}$, it leads to $H_{\left[P, C_{1}\right]}$, which takes the following form

$$
H_{\left[P, C_{1}\right]}=\left[\begin{array}{rr}
-P\left(I-C_{1} P\right)^{-1} C_{1} & P\left(I-C_{1} P\right)^{-1} \\
-\left(I-C_{1} P\right)^{-1} C_{1} & \left(I-C_{1} P\right)^{-1}
\end{array}\right] .
$$

To reveal the connection between the four transfer functions in $H_{\left[P, C_{1}\right]}$ and the transfer functions in Figure 2, we need to define another transfer function in Figure 2 as $D_{i}: \tilde{r}(t) \mapsto y(t)$, that is, the reference-to-output transfer function, which is denoted as $D_{i}:=N\left(\tilde{K}_{i} G\right)^{-1}=P\left(I-C_{i} P\right)^{-1} \tilde{V}_{i}^{-1}$; then, the following result, analogous to that of Lemma 4, can be obtained.

\section{Lemma 9}

If $\left[P, C_{i}\right], i=0,1$ are internally stable, then $D_{1}=D_{0} T^{-1} \in \mathscr{R} \mathscr{H}_{\infty}$.

\section{Proof}

Because $\left[P, C_{i}\right]$ is internally stable, we have $\left(\tilde{K}_{i} G\right)^{-1} \in \mathscr{R} \mathscr{H}_{\infty}$. This together with the fact that $N \in \mathscr{R} \mathscr{H}_{\infty}$ implies that $D_{i}=N\left(\tilde{K}_{i} G\right)^{-1} \in \mathscr{R} \mathscr{H}_{\infty}$. Because $D_{i}=N\left(\tilde{K}_{i} G\right)^{-1}=P W_{i}, i=0,1$, it follows from Lemma 8 that $D_{1}=P W_{1}=P W_{0} T^{-1}=D_{0} T^{-1}$.

We shall now connect the transfer functions in $H_{\left[P, C_{1}\right]}$ and the transfer functions $T, W_{0}, D_{0}$ in Figure 2. We have the following result. 


\section{Theorem 10}

Consider the feedback configuration in Figure 1 and the setting in Figure 2. Suppose that $\left[P, C_{i}\right]$, $i=0,1$ are internally stable. Let $P=N M^{-1}, C_{0}=\tilde{V}_{0}^{-1} \tilde{U}_{0}$, and $C_{1}=\tilde{V}_{1}^{-1} \tilde{U}_{1}$ be coprime factorizations over $\mathscr{R} \mathscr{H}_{\infty}$ and $T: \tilde{r} \mapsto z$ be defined by (6), and let $W_{i}=M\left(\tilde{K}_{i} G\right)^{-1}=\left(I-C_{i} P\right)^{-1} \tilde{V}_{i}^{-1}$, $i=0,1$ and $D_{i}=N\left(\tilde{K}_{i} G\right)^{-1}=P\left(I-C_{i} P\right)^{-1} \tilde{V}_{i}^{-1}, i=0,1$. Then,

1. $H_{\left[P, C_{1}\right]}=\left[\begin{array}{c}D_{1} \\ W_{1}\end{array}\right]\left[\begin{array}{cc}-\tilde{U}_{1} & \tilde{V}_{1}\end{array}\right]=\left[\begin{array}{c}D_{0} T^{-1} \\ W_{0} T^{-1}\end{array}\right]\left[\begin{array}{cc}-\tilde{U}_{1} & \tilde{V}_{1}\end{array}\right] \in \mathscr{R} \mathscr{H}_{\infty}$.

2. If $C_{1}=\tilde{V}_{1}^{-1} \tilde{U}_{1}$ is a normalized lcf, then $\left\|H_{\left[P, C_{1}\right]}\right\|_{\infty}=\left\|\left[\begin{array}{c}D_{0} T^{-1} \\ W_{0} T^{-1}\end{array}\right]\right\|_{\infty}$.

Proof

It is easy to show that

$$
\begin{gathered}
-P\left(I-C_{1} P\right)^{-1} C_{1}=-D_{1} \tilde{U}_{1}, P\left(I-C_{1} P\right)^{-1}=D_{1} \tilde{V}_{1}, \\
-\left(I-C_{1} P\right)^{-1} C_{1}=-W_{1} \tilde{U}_{1},\left(I-C_{1} P\right)^{-1}=W_{1} \tilde{V}_{1} .
\end{gathered}
$$

Using (10), Lemmas 8 and 9, the conclusions of the theorem are now straightforward.

Because $W_{0}, D_{0}$, and $T$ are the transfer functions from $\tilde{r}(t)$ to $u(t), y(t)$ and $z(t)$, respectively, in Figure 2, the results in the aforementioned theorem reveal clearly the underlying connection between the transfer functions in $H_{\left[P, C_{1}\right]}$ implemented as in Figure 1 and the transfer functions $W_{0}$, $D_{0}, T$ in Figure 2. The theorem is important because it has established the theoretical basis for using Figure 2 as an experimental setting to check the closed-loop performance of the controller $C_{1}$ implemented as in Figure 1. Because the frequency responses of $W_{0}, D_{0}$, and $T$ can be obtained through running experiments using Figure 2, the frequency responses of the four transfer functions in $H_{\left[P, C_{1}\right]}$ implemented as in Figure 1 can be computed using Theorem 10 (to within the limits of experimental accuracy), and thus many frequency domain performance measures when using the controller $C_{1}$. For example, one can obtain the closed-loop bandwidth from $D_{1}(j \omega)$ and $b_{\left[P, C_{1}\right]}$ from the frequency response data of all the four transfer functions in $H_{\left[P, C_{1}\right]}$.

\section{Remark 11}

It should be pointed out that to evaluate the closed-loop performance of a controller, we do not always need the full frequency responses. In many cases, only a limited amount of data of $W_{1}(j \omega)$ and $D_{1}(j \omega)$ at a finite number of frequency points will be sufficient to provide sensible performance evaluations. For example, if we find $\rho\left(P, C_{1}, \omega\right)$ is too close to zero at one particular frequency, then we can stop the experiments and conclude that $C_{1}$ will cause some closed-loop performance problems because $b_{\left[P, C_{1}\right]} \leqslant \rho\left(P, C_{1}, \omega\right)$.

\subsection{Connection between $b_{\left[P, C_{1}\right]}$ and $b_{\left[P, C_{0}\right]}$ through $T$}

It is in general not sufficient to draw valid conclusions on the closed-loop performance of $C_{1}$ by looking at fewer than the four transfer functions in $H_{\left[P, C_{1}\right]}$, at least in the multivariable case, although it may suffice in the SISO case and other specialized cases. The measure $b_{\left[P, C_{0}\right]}$ is one measure that does relate to overall performance. In this subsection, we shall propose analysis results connecting $b_{\left[P, C_{1}\right]}$ and $b_{\left[P, C_{0}\right]}$ through $T$.

Theorem 12

Consider the feedback configuration in Figure 1 and the setting in Figure 2. Suppose that $\left[P, C_{i}\right]$, $i=0,1$ are internally stable. Let $C_{0}=\tilde{V}_{0}^{-1} \tilde{U}_{0}$, and $C_{1}=\tilde{V}_{1}^{-1} \tilde{U}_{1}$ be the normalized lcfs over $\mathscr{R} \mathscr{H}_{\infty}$ and $T: \tilde{r} \mapsto z$ be defined by (6). Let $b_{\left[P, C_{0}\right]}$ and $b_{\left[P, C_{1}\right]}$ be defined as in (4). Then,

$$
b_{\left[P, C_{0}\right]}\left\|T^{-1}\right\|_{\infty}^{-1} \leqslant b_{\left[P, C_{1}\right]} \leqslant b_{\left[P, C_{0}\right]}\|T\|_{\infty} .
$$


Proof

Because $C_{i}=\tilde{V}_{i}^{-1} \tilde{U}_{i}, i=0,1$ are normalized lcfs; it follows from the proof of Theorem 10 that

$$
1 / b_{\left[P, C_{i}\right]}=\left\|H_{\left[P, C_{i}\right]}\right\|_{\infty}=\left\|\left[\begin{array}{c}
D_{i} \\
W_{i}
\end{array}\right]\right\|_{\infty} .
$$

Using results in Lemmas 8, 9, and Theorem 10, one obtains

$$
b_{\left[P, C_{1}\right]}=\frac{1}{\left\|\left[\begin{array}{l}
D_{0} T^{-1} \\
W_{0} T^{-1}
\end{array}\right]\right\|_{\infty}} \geqslant \frac{1}{\left\|\left[\begin{array}{l}
D_{0} \\
W_{0}
\end{array}\right]\right\|_{\infty}\left\|T^{-1}\right\|_{\infty}}=b_{\left[P, C_{0}\right]}\left\|T^{-1}\right\|_{\infty}^{-1}
$$

and

$$
b_{\left[P, C_{0}\right]}=\frac{1}{\left\|\left[\begin{array}{l}
D_{0} T^{-1} T \\
W_{0} T^{-1} T
\end{array}\right]\right\|_{\infty}}=\frac{1}{\left\|\left[\begin{array}{l}
D_{1} T \\
W_{1} T
\end{array}\right]\right\|_{\infty}} \geqslant \frac{1}{\left\|\left[\begin{array}{l}
D_{1} \\
W_{1}
\end{array}\right]\right\|_{\infty}\|T\|_{\infty}}=b_{\left[P, C_{1}\right]}\|T\|_{\infty}^{-1} .
$$

Then, (11) follows immediately.

Using $b_{\left[P, C_{1}\right]} \leqslant b_{\left[P, C_{0}\right]}\|T\|_{\infty}$ and noting that $b_{\left[P, C_{0}\right]} \leqslant 1$, it is easy to see that $b_{\left[P, C_{1}\right]}$ will be close to zero if $\|T\|_{\infty}$ is close to zero, and thus, there might be closed-loop performance problems. This observation may make it possible to check the closed-loop performance of $C_{1}$ by only looking at $\|T\|_{\infty}$. Therefore, the results in the theorem provide further theoretical support on why Figure 2 is a good setting for checking the closed-loop performance of a controller.

When $\|T\|_{\infty}$ is not close to zero, the result in the aforementioned theorem might be too conservative to use. To obtain a better result, we look at a pointwise version of Theorem 12. Also, from an experimental point of view, it makes sense to consider testing over a range of frequencies. The pointwise version of Theorem 12 is as follows:

Theorem 13

Suppose the hypotheses of Theorem 12 hold. For $\forall \omega$, there holds

$$
\underline{\sigma}[T(j \omega)] \rho\left(P, C_{0}, \omega\right) \leqslant \rho\left(P, C_{1}, \omega\right) \leqslant \bar{\sigma}[T(j \omega)] \rho\left(P, C_{0}, \omega\right) .
$$

If $P$ is a SISO system, then there holds

$$
\rho\left(P, C_{1}, \omega\right)=|T(j \omega)| \rho\left(P, C_{0}, \omega\right) .
$$

Proof

Because $C_{i}=\tilde{V}_{i}^{-1} \tilde{U}_{i}, i=0,1$ are normalized lcfs, it follows from the proof of Theorem 10 that

$$
\rho\left(P, C_{i}, \omega\right)=1 / \bar{\sigma}\left[H_{\left[P, C_{i}\right]}(j \omega)\right]=1 / \bar{\sigma}\left[\left[\begin{array}{l}
D_{i} \\
W_{i}
\end{array}\right](j \omega)\right] .
$$

Using (15) and Lemmas 8 and 9, one obtains

$$
\begin{aligned}
\rho\left(P, C_{1}, \omega\right) & =1 / \bar{\sigma}\left[\left[\begin{array}{l}
D_{0} T^{-1} \\
W_{0} T^{-1}
\end{array}\right](j \omega)\right] \\
& \geqslant 1 / \bar{\sigma}\left[\left[\begin{array}{l}
D_{0} \\
W_{0}
\end{array}\right](j \omega)\right] \bar{\sigma}\left[T^{-1}(j \omega)\right]=\underline{\sigma}[T(j \omega)] \rho\left(P, C_{0}, \omega\right)
\end{aligned}
$$

and

$$
\begin{aligned}
\rho\left(P, C_{0}, \omega\right) & =\frac{1}{\bar{\sigma}\left[\left[\begin{array}{l}
D_{0} T^{-1} T \\
W_{0} T^{-1} T
\end{array}\right](j \omega)\right]}=\frac{1}{\bar{\sigma}\left[\left[\begin{array}{l}
D_{1} T \\
W_{1} T
\end{array}\right](j \omega)\right]} \\
& \geqslant \frac{1}{\bar{\sigma}\left[\left[\begin{array}{l}
D_{1} \\
W_{1}
\end{array}\right](j \omega)\right] \bar{\sigma}[T(j \omega)]}=\frac{\rho\left(P, C_{1}, \omega\right)}{\bar{\sigma}[T(j \omega)]} .
\end{aligned}
$$

Then, (13) follows from (16) and (17) immediately. 
When $P$ is a SISO system, it follows that $\underline{\sigma}[T(j \omega)]=\bar{\sigma}[T(j \omega)]=|T(j \omega)|$ and thus (14) holds.

The aforementioned theorem implies that the closed-loop system might have performance problems if $\bar{\sigma}[T(j \omega)]$ becomes too close to zero at certain frequency points. This is verified later in the first simulation example. This makes it possible to detect closed-loop performance problems by looking at only a single transfer function $T$. The theorem is less conservative than Theorem 12 as it is pointwise.

Because $T(j \omega), W_{0}(j \omega)$, and $D_{0}(j \omega)$ are experimentally obtainable through carrying out experiments on Figure 2, it follows from Theorem 10 that the frequency responses of all transfer functions in $H_{\left[P, C_{1}\right]}$ can be obtained experimentally. Therefore, $\rho\left(P, C_{1}, \omega\right)$ and thus $b_{\left[P, C_{1}\right]}$ can be computed using their definitions.

Additionally, in the following, we are going to show that for SISO systems, $\rho\left(P, C_{1}, \omega\right)$ and thus $b_{\left[P, C_{1}\right]}$ can be directly computed using only $T(j \omega), W_{0}(j \omega)$, and $D_{0}(j \omega)$.

\section{Theorem 14}

Suppose the hypotheses of Theorem 13 hold and if a simplification arises in that $\left[P, C_{i}\right], i=0,1$ are stable, then

$$
\begin{gathered}
\rho\left(P, C_{0}, \omega\right)=\frac{1}{\sqrt{\left|D_{0}(j \omega)\right|^{2}+\left|W_{0}(j \omega)\right|^{2}}} \\
\rho\left(P, C_{1}, \omega\right)=\frac{1}{\sqrt{\left|D_{1}(j \omega)\right|^{2}+\left|W_{1}(j \omega)\right|^{2}}}=\frac{|T(j \omega)|}{\sqrt{\left|D_{0}(j \omega)\right|^{2}+\left|W_{0}(j \omega)\right|^{2}}} .
\end{gathered}
$$

Proof

The conclusions of the theorem follow from (15) and Lemmas 8 and 9.

\section{Remark 15}

In this section, we assumed that the physical system is implemented as in Figure 1. However, similar analysis can be carried out for the scenario where the physical system is implemented as in Figure 2, and similar results can be obtained.

From the theoretical results obtained in this section, it is obvious that the main focus for performance verification is how to obtain the frequency response data of $T(j \omega), W_{0}(j \omega)$, and $D_{0}(j \omega)$ through experiments on Figure 2. The estimate of the frequency responses of $T, W_{0}$, and $D_{0}$ up to a required frequency can be obtained by using either parametric [26] or nonparametric [27] estimation methods. Furthermore, whatever method is used to obtain the closed-loop measures, being experimental in nature, it will be tolerant of some amount of noise.

\section{NUMERICAL EXAMPLES}

In this section, we shall present two examples to illustrate the practicality of our results in verifying the closed-loop performance before candidate controllers are switched in. In the first example, a simple plant and controller sets are used to show how to setup and utilize our experimental results. In the second example, we apply our proposed results to more practical situations for verifying the closed-loop performance where a flexible robot arm plant and controllers designed using the so-called Windsurfer approach are used.

\subsection{A simple plant and controller example}

Let us consider a second-order plant $P(s)=1 /(s+1)(.1 s+1)$ and suppose its model, a firstorder approximation, $P_{m}(s)=1 /(s+1)$ is available. Let $C_{1}=-1$ and $C_{2}=-100$ be candidate controllers, designed with $P_{m}(s)$ to provide good closed-loop performances. This classical example 
simply and strikingly shows that even if $P(s)$ and $P_{m}(s)$ generate very similar responses in open loop (as shown in Figure 3), their closed-loop performances can be significantly different (as shown in Figure 4).

This, in fact, motivates why our results for verifying the closed-loop performances of $C_{i}$ with $P$ are necessary in practice. Let us consider a simple scenario when $C_{0}=0$ (i.e. $\tilde{U}_{0}=0$ and $\tilde{V}_{0}=1$ ). Replacing $C_{0}$ in (6) with $C_{1}$ and $C_{2}$, we obtain two mapping $T_{1}$ and $T_{2}$, respectively. The simulation results of the estimates for $\min _{\omega}\left|T_{i}(j \omega)\right|$ and $b_{\left[P, C_{i}^{1}\right]}$ are summarized in Table I.

From the aforementioned results, one can conclude that $\left\|T_{i}\right\|_{\infty}$ by itself is not a very good measure of the closed-loop performance. Yet, as we have both $b_{\left[P, C_{2}\right]}$ and $\min _{\omega}\left|T_{2}(j \omega)\right|$ very close to zero, one should be alarmed that using $C_{2}$ might cause a closed-loop performance problem

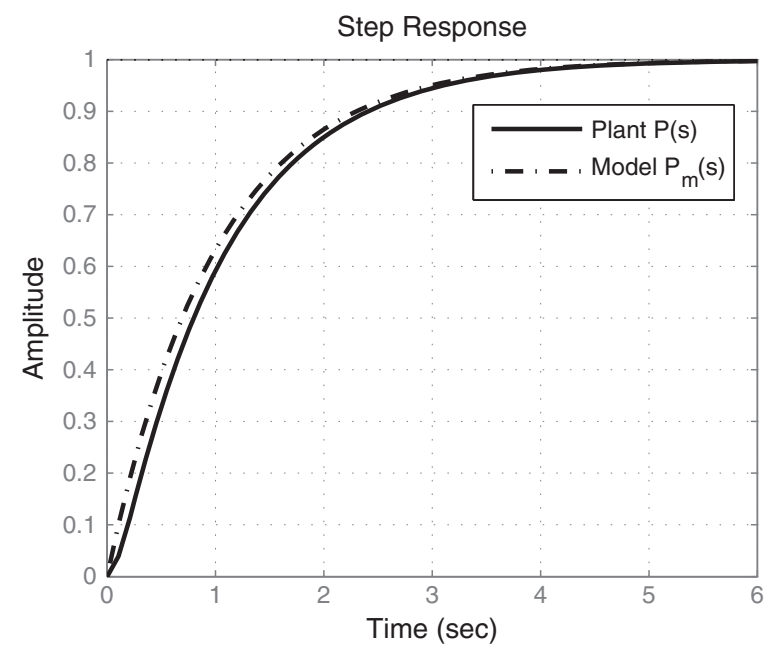

Figure 3. Open loop responses of true plant $P$ and its model $P_{m}$.

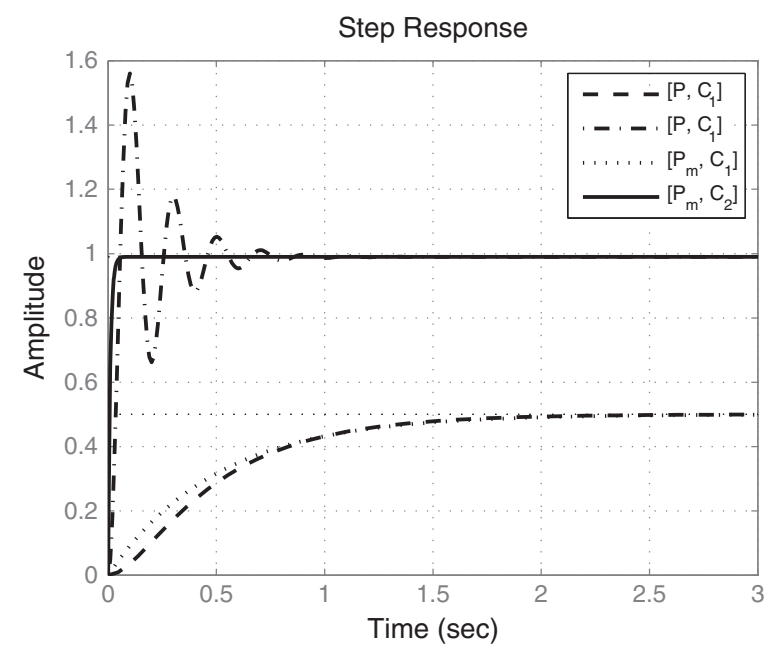

Figure 4. Closed-loop mappings of $r \mapsto y$ for $\left[P, C_{i}\right]$ and $\left[P_{m}, C_{i}\right], i=1,2$.

Table I. Performance measures for $\left[P, C_{i}\right]$.

\begin{tabular}{lccc}
\hline & $\min _{\omega}\left|T_{i}(j \omega)\right|$ & $\left\|T_{i}\right\|_{\infty}$ & $b_{\left[P, C_{i}\right]}$ \\
\hline$\left[P, C_{1}\right]$ & 0.9570 & 2 & 0.6696 \\
{$\left[P, C_{2}\right]$} & 0.0033 & 1.01 & 0.0033 \\
\hline
\end{tabular}




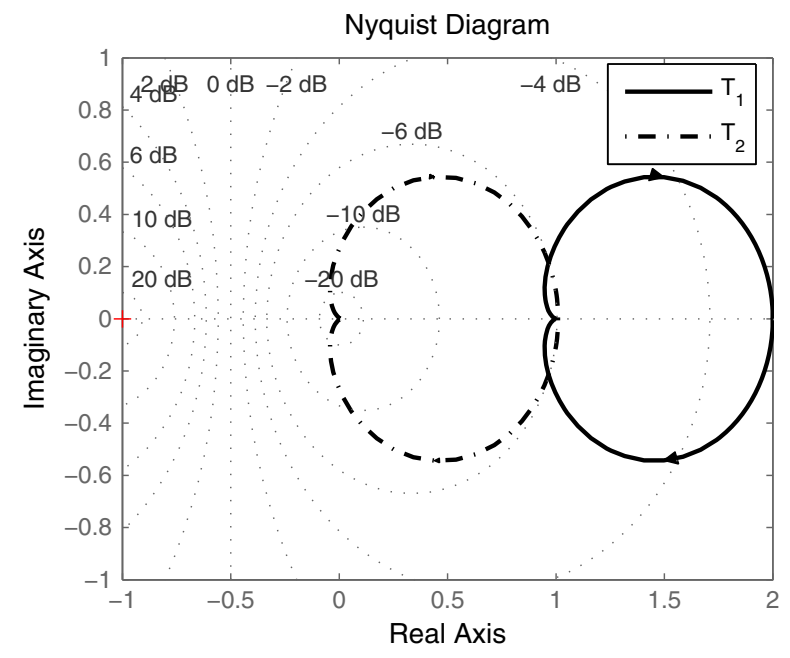

Figure 5. Nyquist diagrams of $T_{1}$ and $T_{2}$.

when it is switched in. In fact, the Nyquist diagrams of $T_{2}$, as shown in Figure 5, indicate that $\left|T_{2}\right|$ gets very close to zero, hence, the performance may be severely degraded.

\subsection{A flexible robot arm example}

The Windsurfer approach is a particular iterative identification and control design procedure, which was successfully used in [2,4,28], and [29] to design controllers to push the closed-loop bandwidth (BW) of a flexible robot arm as large as possible. The ultimate origins of the model are not clear from these references, although the zero and pole structure is quite typical for a flexible robot arm. [30] discusses a very similar model. The basic idea is to start with a rough model, say $P_{0}$ of the plant $P$. Then, one designs a series of controllers $C_{i}^{1}$ on the basis of $P_{0}$, and internal model control (IMC) aiming to push the $\mathrm{BW}$ of $\left[P, C_{i}^{1}\right]$ as far as possible until $\left[P, C_{i}^{1}\right]$ might have performance problems. Then, one re-identifies the plant with the most recent $C_{i}^{1}$ connected to have a better model $P_{1}$ and designs a new series of controllers $C_{i}^{2}$ on the basis of $P_{1}$ and IMC to continue to push the closed-loop BW until $\left[P, C_{i}^{2}\right]$ might have performance problems. Continuing in the same fashion, the design procedure will stop if either a satisfactory controller is found or the given control objectives are manifestly in practice unobtainable with the latest model. In this process, it is desirable to detect the controller that will have performance problems before it is actually inserted into the control loop, and this example shows how to do that. However, it should be pointed out that we will not repeat the entire Windsurfer procedure in this simulation. Instead, we pick up controllers designed in two particular iterations in the Windsurfer procedure to check the performances of those controllers and show how our performance verification results can help the Windsurfer design procedure to decide when plant re-identification is needed or to flag a stop point for the entire procedure.

In our simulations, we shall consider a robot arm plant used in [2,4] and [28]. The plant $P(s)$ is a flexible-link robot arm with transfer function

$$
P(s)=\frac{1.0707(s+13.16)\left(s^{2}-14.34 s+184.6\right)\left(s^{2}+21.29 s+263.9\right)}{\left(s^{2}+0.1992 s+9.02\right)\left(s^{2}+0.6678 s+147.3\right)\left(s^{2}+3.69 s+994.5\right)},
$$

whose parameters are not known to the control system designers.

For this plant, we have a rough model $P_{0}(s)$ (see [2]) as

$$
P_{0}(s)=\frac{0.35175(s+13.31)}{\left(s^{2}+0.1806 s+9.024\right)}
$$

According to the Bode plots shown in Figure 6, it is easy to see that $P_{0}(s)$ captures to a degree the lowest resonance and the roll-off behavior at high frequency. 


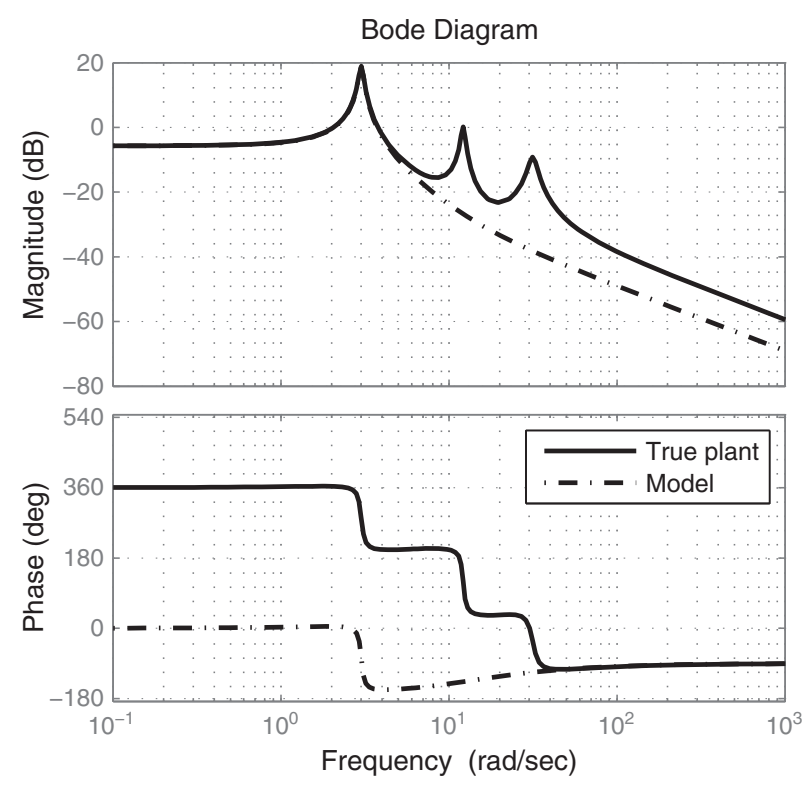

Figure 6. Frequency responses of true plant $P$ and its model $P_{0}$.

On the basis of $P_{0}(s)$, we have designed a controller $C_{0}$ using a standard IMC design method [31] to achieve the closed-loop BW of $0.1 \mathrm{rad} / \mathrm{s}$, which is a stabilizing controller and takes the following form

$$
C_{0}(s)=\frac{-0.28429\left(s^{2}+0.1806 s+9.024\right)}{s(s+13.31)},
$$

which has a normalized lcf as $C_{0}(s)=\tilde{V}_{0}(s)^{-1} \tilde{U}_{0}(s)$ with

$$
\begin{aligned}
U_{0}(s) & =\frac{-0.27346\left(s^{2}+0.1806 s+9.024\right)}{(s+0.1936)(s+12.75)} \\
V_{0}(s) & =\frac{0.96188 s(s+13.31)}{(s+0.1936)(s+12.75)}
\end{aligned}
$$

Because we desire to have the closed-loop BW as large as possible, we design four new controllers $C_{i}^{1}, i \in[1,4]$ aiming to achieve the closed-loop BW of $0.40,0.45,0.50$, and $0.55 \mathrm{rad} / \mathrm{s}$, respectively, using the same IMC methods on the basis of $P_{0}(s)$. The controllers $C_{i}^{1}$ are designed as

$$
C_{i}^{1}(s)=\frac{-K_{i}\left(s^{2}+0.1806 s+9.024\right)}{s(s+13.31)},
$$

where $K_{1}=1.1372, K_{2}=1.2793, K_{3}=1.4215$, and $K_{4}=1.5636$. We have found the normalized lcf factorizations for all these controllers, but do not present them here because of space limitations. As we know $P_{0}(s)$ is only a rough model, we want to be cautious in pushing out the closed-loop BW so that we would like to check the performance of all these four controllers before they are inserted into the control loop.

In the simulations, we first keep $C_{0}$ in the control loop to test the performance of $C_{1}^{1}$ using the measure $b_{\left[P, C_{1}^{1}\right]}$. If $C_{1}^{1}$ performs satisfactorily, then we replace $C_{0}$ in Figure 2 with $C_{1}^{1}$, then go on to test the performance of $C_{2}^{1}$, and so on. If any prospective controller is found to suggest performance problems, then we will stop the testing and insertion of any further remaining controllers, pending re-identification of the plant. To see the effect of noise, a noise process, generated via MATLAB 
Table II. Performance measures for $\left[P, C_{i}^{1}\right]$.

\begin{tabular}{lcccccc}
\hline & \multicolumn{2}{c}{ Analysis } & \multicolumn{2}{c}{ Experiment without noise } & \multicolumn{2}{c}{ Experimental with noise } \\
\cline { 2 - 7 } & $\min _{\omega}|T(j \omega)|$ & $b_{\left[P, C_{i}^{1}\right]}$ & $\min _{\omega}|T(j \omega)|$ & $b_{\left[P, C_{i}^{1}\right]}$ & $\min _{\omega}|T(j \omega)|$ & $b_{\left[P, C_{i}^{1}\right]}$ \\
\hline$\left[P, C_{1}^{1}\right]$ & 0.2862 & 0.1184 & 0.2862 & 0.1183 & 0.2918 & 0.1199 \\
{$\left[P, C_{2}^{1}\right]$} & 0.6593 & 0.1078 & 0.6594 & 0.1080 & 0.6639 & 0.1085 \\
{$\left[P, C_{3}^{1}\right]$} & 0.5293 & 0.0571 & 0.5294 & 0.0574 & 0.5356 & 0.0578 \\
{$\left[P, C_{4}^{1}\right]$} & 0.1848 & 0.0107 & 0.2041 & 0.0121 & 0.2203 & 0.0131 \\
\hline
\end{tabular}

using a standard Gaussian distribution with zero mean and standard deviation 0.05 is added to the output measurements in all the relevant experiments in this section. The particular tests are all spot frequency sine wave tests, with the external input signal $\tilde{r}(t)$ of unit amplitude. The simulation results of the estimates for $\min _{\omega}\left|T_{i}(j \omega)\right|$ and $b_{\left[P, C_{i}^{1}\right]}$ are summarized in Table II.

From the table, it can be observed that our algorithm has provided accurate estimates for $\min _{\omega}|T(j \omega)|$ and $b_{\left[P, C_{i}^{1}\right]}, i=1,2,3,4$. Because $b_{\left[P, C_{4}^{1}\right]}$ is smaller than $b_{\left[P, C_{1}^{1}\right]}$ by a factor of 10 and close to zero, it is likely that $C_{4}^{1}$ will experience closed-loop problems. Although $\min _{\omega}|T(j \omega)|$ for $\left[P, C_{4}^{1}\right]$ has been decreased significantly compared with the value for $\left[P, C_{3}^{1}\right]$ (by a factor of two), it is not decreased much compared with the value for $\left[P, C_{1}^{1}\right]$. This is mainly due to the fact that $\min _{\omega}|T(j \omega)|$ is a performance measure of $\left[P, C_{i}^{1}\right]$ on the basis of the current controller in the closed-loop (i.e. a relative performance measure). Hence, one can conclude that $b_{\left[P, C_{i}^{1}\right]}$ (i.e. an absolute performance measure) is a more revealing performance measure one should use. Now, according to the aforementioned performance verification results, $C_{4}^{1}$ should not be put into the control loop, and it should be safer to keep $C_{3}^{1}$ in the control loop. This means that we should not use model $P_{0}$ to push the closed-loop BW beyond $0.50 \mathrm{rad} / \mathrm{s}$. This result is consistent with the conclusions of a simple analysis using knowledge of $P(s)$, which is not available to the designer; it is found that the $\left[P, C_{4}^{1}\right]$ has very large resonances (up to $33 \mathrm{~dB}$ for mapping $r \mapsto u$ ) when compared with the moderate resonances with $\left[P, C_{3}^{1}\right]$ (about $18 \mathrm{~dB}$ for mapping $r \mapsto u$ ), as shown in Figure 7, and any controller with the goal of achieving the closed-loop BW beyond $0.57 \mathrm{rad} / \mathrm{s}$ will actually be destabilizing.

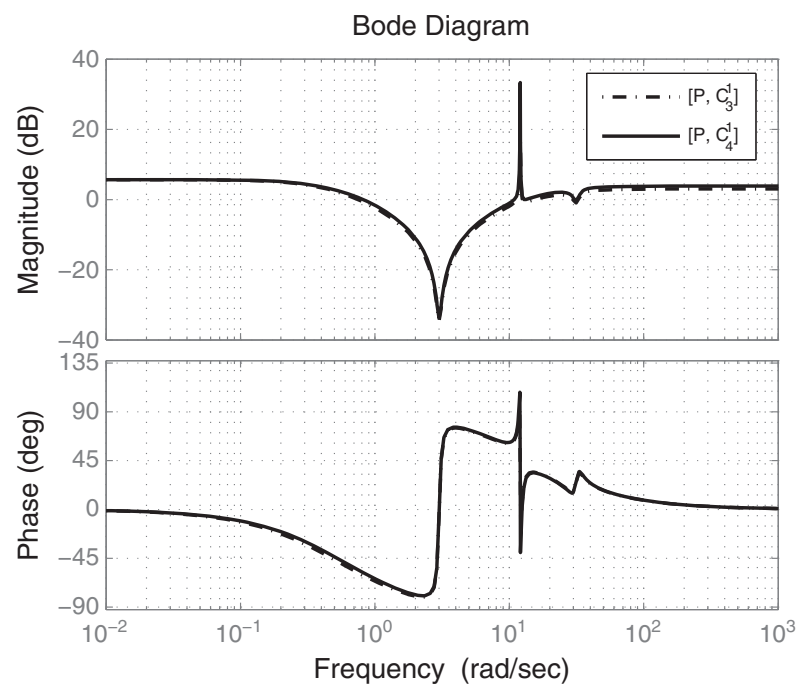

Figure 7. Bode plots of the mapping $r \mapsto u$ for $\left[P, C_{3}^{1}\right]$ (broken line) and $\left[P, C_{4}^{1}\right]$ (solid line). 
We have also run simulations to check the closed-loop BW that $C_{3}^{1}$ can actually achieve with $C_{2}^{1}$ in the control loop. Because we have some information about the closed-loop BW $\left(C_{3}^{1}\right.$ is intended to have the closed-loop BW of $0.50 \mathrm{rad} / \mathrm{s}$ ), our search for the closed-loop BW of $\left[P, C_{3}^{1}\right]$ was carried over a finite grid of frequencies from 0.1 to $1.0 \mathrm{rad} / \mathrm{s}$ with an increment of $0.01 \mathrm{rad} / \mathrm{s}$. The simulation results are summarized in Table III.

It can be observed from Table III that our algorithm can provide adequate estimates for the considered measures even with noisy data. It is not surprising to see that the closed-loop BW of $\left[P, C_{3}^{1}\right]$ is not significantly different from the nominated closed-loop BW of $0.50 \mathrm{rad} / \mathrm{s}$ as $P$ is not significantly different from $P_{0}$ up to around $5 \mathrm{rad} / \mathrm{s}$.

Now, suppose that it is desirable that the closed-loop BW be expanded to be as large as possible. Because we have found out that with the model $P_{0}$, the closed-loop BW can only be safely pushed to around $0.50 \mathrm{rad} / \mathrm{s}$; a new iteration of model identification and control is required at this point because a better model is needed for the greater closed-loop BW. In [2], a better model was identified as

$$
P_{1}(s)=\frac{1.3005(s+12.97)\left(s^{2}+14.67 s+175.9\right)\left(s^{2}-18.2 s+228.5\right)}{\left(s^{2}+0.179 s+9.024\right)\left(s^{2}+0.9668 s+145\right)\left(s^{2}+4.95 s+998.5\right)}
$$

The Bode plots shown in Figure 8 confirm that it is indeed a much better model.

Table III. Analytical and experimental performance for $\left[P, C_{3}^{1}\right]$.

\begin{tabular}{lccc}
\hline & Analysis & Experiment without noise & Experimental with noise \\
\hline DC-gain & 1.0000 & 1.0010 & 0.8797 \\
Cut-off mag & 0.7070 & 0.7077 & 0.6219 \\
BW (rad/s) & 0.5018 & $\approx 0.502$ & $\approx 0.503$ \\
\hline
\end{tabular}

BW, bandwidth.

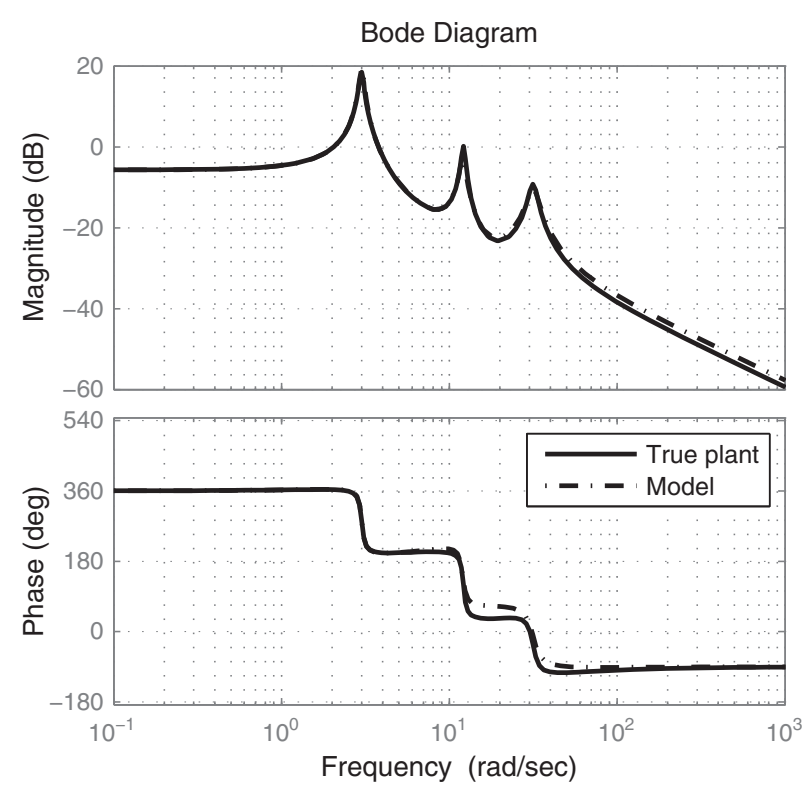

Figure 8 . Frequency responses of true plant $P$ and its model $P_{1}$. 
On the basis of $P_{1}(s)$, we again design four controllers $C_{i}^{2}, i \in[1,4]$ aiming to achieve the closed-loop bandwidth of 2, 4, 8, and $16 \mathrm{rad} / \mathrm{s}$ using the standard IMC design methods as follows:

$$
\begin{aligned}
& C_{1}^{2}(s)=\frac{-6.7214\left(s^{2}+0.179 s+9.024\right)\left(s^{2}+0.9668 s+145\right)\left(s^{2}+4.95 s+998.5\right)}{(s+12.97)(s+1.527 e-006)\left(s^{2}+14.67 s+175.9\right)\left(s^{2}-1.791 s+1167\right)}, \\
& C_{2}^{2}(s)=\frac{-13.4428\left(s^{2}+0.179 s+9.024\right)\left(s^{2}+0.9668 s+145\right)\left(s^{2}+4.95 s+998.5\right)}{(s+12.97)(s+2.668 e-006)\left(s^{2}+14.67 s+175.9\right)\left(s^{2}-8.532 s+1336\right)}, \\
& C_{3}^{2}(s)=\frac{-26.8856\left(s^{2}+0.179 s+9.024\right)\left(s^{2}+0.9668 s+145\right)\left(s^{2}+4.95 s+998.5\right)}{(s+12.97)(s+4.259 e-006)\left(s^{2}+14.67 s+175.9\right)\left(s^{2}-22.01 s+1674\right)}, \\
& C_{4}^{2}(s)=\frac{-53.7713\left(s^{2}+0.179 s+9.024\right)\left(s^{2}+0.9668 s+145\right)\left(s^{2}+4.95 s+998.5\right)}{(s+12.97)(s+6.069 e-006)\left(s^{2}+14.67 s+175.9\right)\left(s^{2}-48.98 s+2350\right)} .
\end{aligned}
$$

We now want to check the closed-loop performance of these four controllers using the scheme of Figure 2. Again here, we are interested in measuring $b_{\left[P, C_{i}^{2}\right]}$ using a limited number of experiments. In the simulations, we first keep $C_{3}^{1}$ in the control loop to test the performance of $C_{1}^{2}$ using the measure $b_{\left[P, C_{1}^{2}\right]}$. If the test with $C_{1}^{2}$ indicates satisfactory performance, then we replace $C_{3}^{1}$ in Figure 2 with $C_{1}^{2}$, then go on to test the performance of $C_{2}^{2}$, and so on. If any controller is found to have performance problems, then we will stop the testing of any further remaining controllers. The simulation results of the estimates for $\min _{\omega}\left|T_{i}(j \omega)\right|$ and $b_{\left[P, C_{i}^{1}\right]}$ are summarized in Table IV.

It can be observed that $b_{\left[P, C_{3}^{2}\right]}$ is very small. Therefore, one should be cautious about using $\left[P, C_{3}^{2}\right]$. The value of $b_{\left[P, C_{4}^{2}\right]}$ is around 0.0065 (with and without noise), which is too small. On the basis of these simulation results, one may use $\left[P, C_{3}^{2}\right]$ to have an aggressive design. However, $\left[P, C_{4}^{2}\right]$ should be avoided. Similar to the performance measurements for $\left[P, C_{i}^{1}\right]$, the aforementioned results for $\left[P, C_{i}^{2}\right]$ also confirm that $b_{\left[P, C_{i}^{2}\right]}$ is still a better performance measure than $\min _{\omega}|T(j \omega)|$.

To check further whether our verification results can provide a valid prediction of closed-loop performance, one can look at the Bode plots of $H_{\left[P, C_{3}^{2}\right]}$ and $H_{\left[P, C_{4}^{2}\right]}$. In fact, we have identified an extremely high gain in the mapping $r \mapsto u$ in $H_{\left[P, C_{4}^{2}\right]}$, as shown in Figure 9, which indicates a problems in the closed-loop performance.

In summary, the first example shows that the potential closed-loop performance problem can be detected for some systems by only looking at one transfer function $T$. The second example demonstrates that $T$ alone may not be a good indicator, whereas the performance tests using the measure $b_{[P, C]}$ worked particularly well in terms of flagging the performance problems of those controllers designed via the Windsurfer approach.

Table IV. Performance measures for $\left[P, C_{i}^{2}\right]$.

\begin{tabular}{lcccccc}
\hline & \multicolumn{2}{c}{ Analysis } & \multicolumn{2}{c}{ Experiment without noise } & \multicolumn{2}{c}{ Experimental with noise } \\
\cline { 2 - 7 } & $\min _{\omega}|T(j \omega)|$ & $b_{\left[P, C_{i}^{2}\right]}$ & $\min _{\omega}|T(j \omega)|$ & $b_{\left[P, C_{i}^{2}\right]}$ & $\min _{\omega}|T(j \omega)|$ & $b_{\left[P, C_{i}^{2}\right]}$ \\
\hline$\left[P, C_{1}^{2}\right]$ & 0.1976 & 0.1181 & 0.1975 & 0.1182 & 0.2038 & 0.1191 \\
{$\left[P, C_{2}^{2}\right]$} & 0.3817 & 0.0487 & 0.3817 & 0.0487 & 0.3847 & 0.0488 \\
{$\left[P, C_{3}^{2}\right]$} & 0.3189 & 0.0175 & 0.3189 & 0.0177 & 0.3221 & 0.0180 \\
{$\left[P, C_{4}^{2}\right]$} & 0.2657 & 0.0064 & 0.2657 & 0.0065 & 0.2660 & 0.0065 \\
\hline
\end{tabular}




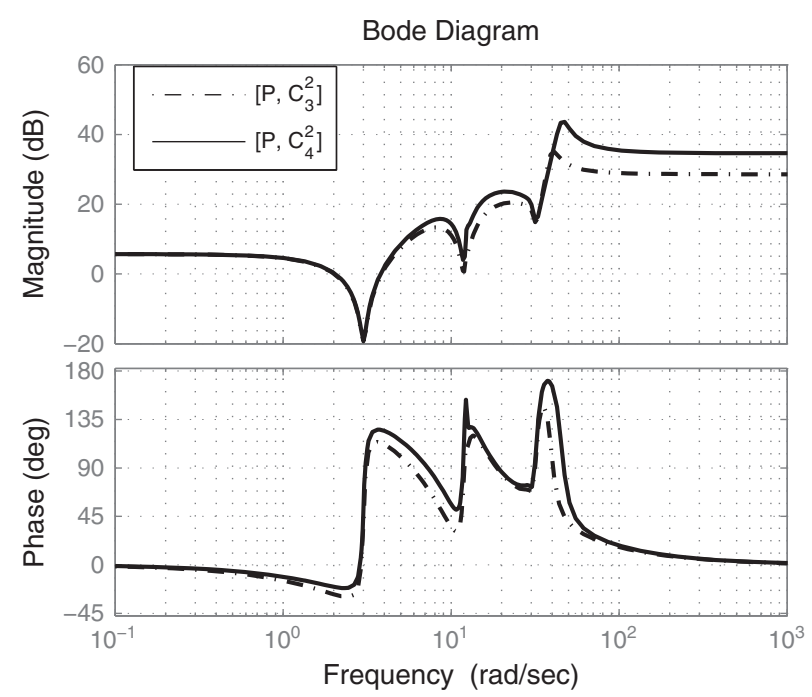

Figure 9. Bode plots of the mapping $r \mapsto u$ for $\left[P, C_{3}^{2}\right]$ (dotted) and $\left[P, C_{4}^{2}\right]$ (solid).

\section{CONCLUSIONS}

In this paper, we have proposed novel closed-loop performance analysis tools and experimental tests. The analysis tools have established the connection between the four closed-loop transfer functions and the transfer functions in the experimental setting in Figure 2. On the basis of these analysis results, tests have been proposed to experimentally carry out the verification of the closed-loop performance of a newly design controller without inserting it into the actual control loop. The results in this paper have extended the results presented in Theorem 7 of [12] from checking only the closed-loop stability to verifying further the closed-loop performance using Figure 2.

To illustrate how the closed-loop performance verification results can be applied in practice, we have applied them to check the closed-loop performance of the controllers designed using the Windsurfer approach. The simulation results on a flexible-link robot arm plant are promising in the sense that the tests proposed in this paper can predict accurately: the closed-loop performance of candidate controllers can be estimated and thus be able to signal potential performance problems with the plant effectively in advance. In addition, our results can be used to suggest a point where the Windsurfer approach requires a model re-identification (before the actual connection of a controller yielding poor closed-loop performance). The simulation results demonstrate that the closed-loop performance verification results are very useful in preventing the Windsurfer approach from connecting a controller possessing some closed-loop performance problems.

As already seen in our simulation results, our results have potential to be used in iterative identification and control. Future research topics may include developing performance tests for more complicated systems such as time-varying systems and nonlinear systems.

\section{ACKNOWLEDGEMENT}

This work was supported in part by the ARC Discovery-Projects Grant DP0664427.

\section{REFERENCES}

1. Lee WS, Anderson BDO, Kosut RL, Mareels IMY. A new approach to adaptive robust control. International Journal of Adaptive Control and Signal Processing 1993; 7:183-211.

2. Lee WS, Anderson BDO, Mareels IMY, Kosut RL. On some key issues in the windsurfer approach to adaptive robust control. Automatica 1995; 11:1169-1636.

3. Hjalmarsson H, Gevers M, Gunnarson S, Lequin O. Iterative feedback tuning: theory and applications. The IEEE Control Systems Magazine 1998; 18(4):26-41. 
4. Anderson BDO. Windsurfing approach to iterative control design. In Iterative Identification and Control, Albertos P, Sala A (eds). Springer-Verlag: London, Great Britain, 2002; 143-166.

5. Gevers M. Identification for control: from the early achievements to the revival of experiment design. European Journal of Control 2005; 11(4-5):335-352.

6. Morse AS. Supervisory control of families of linear set-point controller part i. Exact matching. IEEE Transactions on Automatic Control 1996; 41(10):1413-1431.

7. Narendra KS, Balakrishnan J. Adaptive control using multiple models. IEEE Transactions on Automatic Control 1997; 42:171-187.

8. Anderson BDO, Brinsmead T, De Bruyne F, Hespanha J, Liberzon D, Morse AS. Multiple model adaptive control. Part 1: finite controller coverings. International Journal of Robust and Nonlinear Control 2000; 10(11-12):909-929.

9. Hespanha J, Liberzon D, Morse AS, Anderson BDO, Brinsmead T, De Bruyne F. Multiple model adaptive control Part 2: switching. International Journal of Robust and Nonlinear Control 2001; 11(5):479-496.

10. Anderson BDO. Two decades of adaptive control pitfalls. Proceedings of 8th International Conference on Control, Automation, Robotics and Vision, China, 2004; 1-8.

11. Anderson BDO. Failures of adaptive control theory and their resolution. Communications in Information and Systems 2005; 5-1:1-20.

12. Dehghani A, Anderson BDO, Lanzon A, Lecchini-Visintini A. Verifying stabilizing controllers via closed-loop noisy data: MIMO case. Proceedings of the 46th IEEE Conference on Decision and Control, New Orleans, LA, USA, 2007; 264-269.

13. Dehghani A, Anderson BDO, Lanzon A. Unfalsified adaptive control: a new controller implementations and some remarks. Proceedings of the 2007 European Control Conference, Kos, Greece, 2007; 709-716.

14. Engell S, Tometzki T, Wonghong T. A new approach to adaptive unfalsified control. Proceedings of the 2007 European Control Conference, Kos, Greece, 2007; 1328-1333.

15. Anderson BDO, Gevers MR. Fundamental Problems in Adaptive Control, Perspectives in Control. Springer-Verlag: Berlin, 1998

16. de Callafon RA, Van den Hof PMJ. Suboptimal feedback control by a scheme of iterative identification and control design. Mathematical Modelling of Systems 1997; 13:77-101.

17. Gevers MR, Bombois X, Codrons B, De Bruyne F, Scorletti G. The Role of Experimental Conditions in Model Validation for Control, Robustness in Identification and Control, Vol. 245. Springer Verlag: London, Great Britain, 1999.

18. Anderson BDO, Dehghani A. Historical, generic and current challenges of adaptive control. Proceedings of 9th IFAC Workshop on Adaptation and Learning in Control and Signal Processing, ALCSOP 07, St Petersburg, Russia, 2007; $1-12$.

19. Dehghani A, Lecchini-Visintini A, Lanzon A, Anderson BDO. Validating controllers for internal stability utilizing closed-loop data. IEEE Transactions on Automatic Control 2009; 54:2719-2725.

20. Dehghani A, Cha SH, Anderson BDO. Verifying closed-loop performance before inserting a new controller. Proceedings of the 2010 American Control Conference (ACC'10), Baltimore, Maryland, USA, 2010; 4022-4027.

21. Zhou K, Doyle JC. Essentials of Robust Control. Prentice-Hall, 1997.

22. Linard N, Anderson BDO, De Bruyne F. Identification of a nonlinear plant under nonlinear feedback using left coprime fractional based representation. Automatica 1999; 335(4):655-668.

23. Vinnicombe G. Uncertainty and Feedback: $\mathscr{H}_{\infty}$ Loop-Shaping and the v-Gap Metric. Imperial College Press: London, Great Britain, 2000.

24. Anderson BDO, Dehghani A. Challenges of adaptive control past, permanent and future. Annual Reviews in Control 2008; 32:123-135.

25. Mitra SK. Digital Signal Processing: A Computer Based Approach, (3rd edn). McGraw-Hill, 2005.

26. Ljung L. System Identification Theory for the Users, (2nd edn). Prentice Hall: Upper Saddle River, N.J., 1999.

27. Pintelon R, Schoukens J. System Identification: A Frequency Domain Approach. Wiley-IEEE Press: New York, 1979.

28. Dehghani A, Lanzon A, Anderson BDO. The windsurfer approach to interactive control and identification using an $h_{\infty}$ algorithm. IFAC Workshop on Adaption and Learning in Control and Signal Processing ALCOSP04, Yokohama, Japan, 2004; 777-782.

29. Dehghani A, Lanzon A, Anderson BDO. An $h_{\infty}$ algorithm for the windsurfer approach to adaptive robust control. International Journal of Adaptive Control and Signal Processing 2004; 18:607-628.

30. Pota HR. A prototype flexible robot arm - an interdisciplinary undergraduate project. IEEE Transactions on Education 1992; 35:83-89.

31. Morari M, Zafiriou E. Robust Process Control. Prentice-Hall: Englewood Cliffs, NJ, 1989. 